\title{
ESTUDO CINÉTICO DA REDUÇÃO DE FINOS DE MINÉRIO DE MANGANÊS PELO CARBONO SÓLIDO
}

\author{
José Roberto de Oliveira ${ }^{1}$ \\ Ramiro da Conceição Nascimento ${ }^{2}$ \\ Estéfano Aparecido Vieira ${ }^{3}$ \\ Jorge Alberto Soares Tenório ${ }^{4}$
}

\begin{abstract}
Resumo: Neste trabalho, o $\mathrm{MnO}_{2}$ contido em finos de minério de manganês é reduzido somente pelo carbono grafite sólido presente em uma escória formada pela adição destes finos em um banho Fe-C-Mn. Para isto, foi elaborado uma mistura com finos de grafite e finos de minério, e esta mistura foi carregada em um banho de Fe-C-Mn com $60 \% \mathrm{Mn}$, o que garantiu não ocorresse a redução pelo carbono dissolvido. Os ensaios foram realizados nas temperaturas de $1600^{\circ} \mathrm{C}, 1550^{\circ} \mathrm{C}$ e $1500^{\circ} \mathrm{C}$ e foram usados três tamanhos de partículas de grafite a saber: $0,057 \mathrm{~mm}, 0,162 \mathrm{~mm}$ e $0,230 \mathrm{~mm}$. Foi constatado que todo $\mathrm{FeO}$ foi reduzido a ferro metálico, e que todo $\mathrm{MnO}_{2}$ foi reduzido a $\mathrm{MnO}$ antes de cinco minutos de reação. Em cada caso foi determinada a velocidade da reação de redução do $\mathrm{MnO}$, sendo que as velocidades iniciais encontradas respectivamente foram de $8,74 \times 10^{-2} \mathrm{~mol} / \mathrm{min}$, $6,30 \times 10^{-2} \mathrm{~mol} / \mathrm{min}$ e $5,53 \times 10^{-2} \mathrm{~mol} / \mathrm{min}$. Estes resultados levam a concluir que a velocidade de redução do $\mathrm{MnO}$ é inversamente proporcional ao tamanho das partículas de grafite. Foi também determinada a energia de ativação aparente da reação e o valor encontrado foi de $311,3 \mathrm{~kJ} / \mathrm{mol}$, que confirma que o controle da reação é feito através da reação de Boudouard $\left(\mathrm{CO}_{2(\mathrm{~g})}+\mathrm{C}_{(\mathrm{s})}=2 \mathrm{CO}_{(\mathrm{g})}\right)$
\end{abstract}

Palavras chaves: cinética de redução, minério de manganês, carbono sólido

Abstract: In this study, the $\mathrm{MnO}_{2}$ contained in manganese ores fines is reduced only by solid carbon graphite present in a slag formed by addition of these fines in a Fe-C-Mn bath. In order to this, it was elaborated a mixture of fines of graphite and ore, fines and this mixture was loaded in a $60 \% \mathrm{Mn}$ Fe-C-Mn bath, which guaranteed that the reduction by dissolved carbon did not happen. The experiments were performed in a temperature of $1600^{\circ} \mathrm{C}, 1550^{\circ} \mathrm{C}$ and $1500^{\circ} \mathrm{C}$ and three sizes graphite particles were employed: $0.057 \mathrm{~mm}$; $0.162 \mathrm{~mm}$ and $0.230 \mathrm{~mm}$. It was observed that all $\mathrm{FeO}$ was reduced to metallic iron, and that all $\mathrm{MnO}_{2}$ was reduced to $\mathrm{MnO}$ before five minutes of reaction. In each case, it was determined the speed reaction of $\mathrm{MnO}$ reduction and the initial rate found were of $8,74 \times 10^{-2} \mathrm{~mol} / \mathrm{min}, 6,30 \times 10^{-2} \mathrm{~mol} / \mathrm{min}$ and $5,53 \times 10^{-2} \mathrm{~mol} / \mathrm{min}$, respectively, which permit conclude that the rate of the $\mathrm{MnO}$ reduction is inversely proportional to the size of the graphite particles. Also, the apparent activation energy of the reaction was determined, and it corresponded to $311,3 \mathrm{~kJ} / \mathrm{mol}$, which confirms the control of the reaction is done by Boudouard reaction $\left(\mathrm{CO}_{2(\mathrm{~g})}+\mathrm{C}_{(\mathrm{s})}=2 \mathrm{CO}_{(\mathrm{g})}\right)$

Key Words: Kinetics of reduction; manganese ore; solid carbon

\footnotetext{
${ }^{1}$ CEFETES- Coordenadoria de Metalurgia e Materiais, jroberto@cefetes.br

${ }^{2}$ CEFETES- Coordenadoria de Metalurgia e Materiais, ramiro@cefetes.br

${ }^{3}$ CEFETES- Coordenadoria de Metalurgia e Materiais, estefanovieira@cefetes.br

${ }^{4}$ USP- Departamento de Eng. Metalúrgica e Matérias, jtenorio@usp.br
} 


\section{INTRODUÇÃO}

O processo de Fusão-Redução [1 a 16] foi desenvolvido inicialmente, com base na redução dos óxidos pelo carbono dissolvido no banho. Porém, à medida que o processo foi implantado industrialmente, para a fabricação de ferro-gusa e aço, os estudos mostraram que a redução dos óxidos ocorria em grande parte pelo carbono sólido presente na escória.

$\mathrm{Xu}$ Kuangdi et al [17] realizaram um estudo da redução do $\mathrm{MnO}$ em escórias, voltado para produção de ferro manganês pelo processo de fusão-redução.

Segundo os autores, aumentando-se o teor de manganês inicial do banho diminui-se a taxa de redução do $\mathrm{MnO}$, o que ocorre de acordo com a seguinte equação global:

$$
(\mathrm{MnO})+\underline{\mathrm{C}}=\underline{\mathrm{Mn}}+\mathrm{CO}_{(\mathrm{g})}
$$

Katayama et al $[9,10]$, estudaram a redução de sínter de minério de manganês em forno de indução com capacidade de 100 e $1000 \mathrm{~kg}$. Os autores realizaram também experiências para estudar a redução do $\mathrm{MnO}$ em escória, por uma liga de Fe-Mn saturada em carbono, através da observação da evolução de CO por meio de raios-X. Foram usados banhos com os teores de Mn de 7\%, 22\% e 50\%.

Para o ensaio feito com $7 \%$ de $\mathrm{Mn}$, observou-se a evolução de bolhas de CO, devido a redução do $\mathrm{MnO}$ pelo carbono dissolvido. Esta evolução diminuiu para o ensaio utilizando o teor de $22 \%$ de Mn. Já no terceiro caso, onde o teor de $\mathrm{Mn}$ no banho foi de $50 \%$, não houve evolução de $\mathrm{CO}$, o que indica que não houve redução.

Baseado nestes resultados, o autores afirmam que a redução do $\mathrm{MnO}$ diminui com o aumento na concentração de manganês no banho, e em um processo de fusão-redução para ligas de $\mathrm{Fe}-\mathrm{Mn}$ a maioria das reações de redução ocorrem na interface escória-carvão.

Oliveira $[4,5,18]$ mostrou que para uma liga Fe-Mn-C com teores de Mn no banho em torno de $40 \%$, fazem com que a ENGEVISTA, v. 10, n. 2, p. 82-90 dezembro 2008 redução do $\mathrm{MnO}$ pelo carbono dissolvido se torne muito lenta, a ponto de poder ser desconsiderada.

Porém poucos trabalhos foram feitos no sentido de investigar a redução de óxidos de manganês pelo carbono sólido.

Akdogan e Eric [19] estudaram a redução de minério de manganês pelo carbono sólido, em temperaturas de 1100 a $1350^{\circ}$ C. Os autores afirmam que a redução dos óxidos de manganês ocorre em duas etapas, sendo reduzidos a $\mathrm{MnO}$ antes de 5 minutos. Constataram também que a velocidade desta redução, aumenta com o aumento da temperatura. A energia de ativação aparente para a etapa rápida, que é a redução dos óxidos superiores a $\mathrm{MnO}$, está entre 81 a $94 \mathrm{~kJ} / \mathrm{mol}$. Para a etapa de redução do $\mathrm{MnO}$ pelo carbono dissolvido, ou pelo carbono sólido, foram encontrados valores entre 102 a 141,7 kJ/mol, e foi considerado que o mecanismo controlador é a reação química, na interface $\mathrm{MnO} /$ carbono sólido, ou $\mathrm{MnO} /$ carbono dissolvido. Já Rankin e Wynnyckyj ${ }^{[20]}$ estudaram a redução do $\mathrm{MnO}$ pelo grafite na faixa de temperatura de $1227^{\circ} \mathrm{C}$ a $1427^{\circ} \mathrm{C}$ e encontraram valores de energia de ativação na faixa de 240 a $257 \mathrm{~kJ} / \mathrm{mol}$ que são característicos de processos de redução controlados pela reação de Boudouard.

\section{OBJETIVOS}

Os objetivos deste trabalho são determinar a velocidade de redução do $\mathrm{MnO}$ pelo carbono sólido (grafite) contido em escória, assim como a influência da tamanho das partículas de grafite e da temperatura nesta velocidade, e determinar o mecanismo controlador da reação.

\section{METODOLOGIA}

Para evitar que houvesse redução do $\mathrm{MnO}$, pelo carbono dissolvido no banho, foi usada uma liga de Fe-Mn com teor de Mn igual a $60 \%$, que de acordo com a literatura $[4,5,9,10]$, evita que esta reação ocorra por motivos cinéticos. 
O banho metálico de Fe-Mn saturado em carbono, foi carregado em um cadinho de alumina.

Os cadinhos de alumina foram colocados dentro de cadinhos de grafite, com um diâmetro interno de $5 \mathrm{~cm}$ e altura de $13 \mathrm{~cm}$. Este procedimento foi tomado, para evitar um possível vazamento do banho, caso a cadinho de alumina trincasse por algum motivo. Em nenhum dos ensaios este fato ocorreu. Depois do carregamento do minério para dentro do cadinho, que já estava no interior do forno. A queda na temperatura foi então de $30^{\circ} \mathrm{C}$, o que foi corrigido aumentando-se a temperatura do forno antes do carregamento $\mathrm{O}$ minério carregado não foi totalmente reduzido, sobrando certa quantidade de escória contendo $\mathrm{MnO}$ em cada ensaio.

A figura 1, a seguir, mostra um esquema do forno usado.

As dimensões dos cadinhos de alumina, foram as seguintes:

-altura externa: $10,0 \mathrm{~cm}$

-altura interna: $9,7 \mathrm{~cm}$

-diâmetro externo: 4,6cm

-diâmetro interno: $4,0 \mathrm{~cm}$

O banho foi preparado com os seguintes materiais:

- ferro em pó, com pureza acima de 99,9\% da marca Nuclear,
- manganês eletrolítico, com pureza acima de $99,9 \%$ da marca Nuclear,

- carbono grafite em pó, fornecido pela Egyper com pureza de 99,9\%:

A quantidade de banho usada nestes ensaios foi de 200 gramas.

O carregamento dos finos de minério de manganês e do carbono foi feito de modo que se obtivesse uma distribuição o mais uniforme possível do carbono na escória, e que todo carbono estivesse sempre dentro da camada de escória. Por isto, minério e grafite foram divididos em três partes, que foram carregadas alternadamente. Durante os ensaios não foi observada a segregação de grafite para a superfície do banho.

A quantidade de minério foi estipulada em $150 \mathrm{~g}$ e sua granulometria $100 \%$ abaixo de $0,20 \mathrm{~mm}$.

A tabela 1 mostra a análise química em base seca do minério usado.

Tabela 1. Composição química do fino de minério de Mn usado.

\begin{tabular}{ccccc}
\hline $\mathbf{M n} *$ & $\mathbf{F e}^{*}$ & $\mathbf{S i O}_{2}$ & $\mathbf{A l}_{2} \mathbf{O}_{3}$ & $\mathbf{P}$ \\
\hline 42,3 & 7,8 & 9,1 & 12,8 & 0.07 \\
\hline$* \mathbf{M n}$ na forma de $\mathbf{M n O}_{2} \mathbf{e ~ F e}$ na forma de $\mathbf{F e}_{2} \mathbf{O}_{3}$
\end{tabular}

Porém, como será mostrado adiante, todo $\mathrm{MnO}_{2}$ é reduzido a $\mathrm{MnO}$ antes dos primeiros 5 minutos de reação, e todo tratamento então será feito

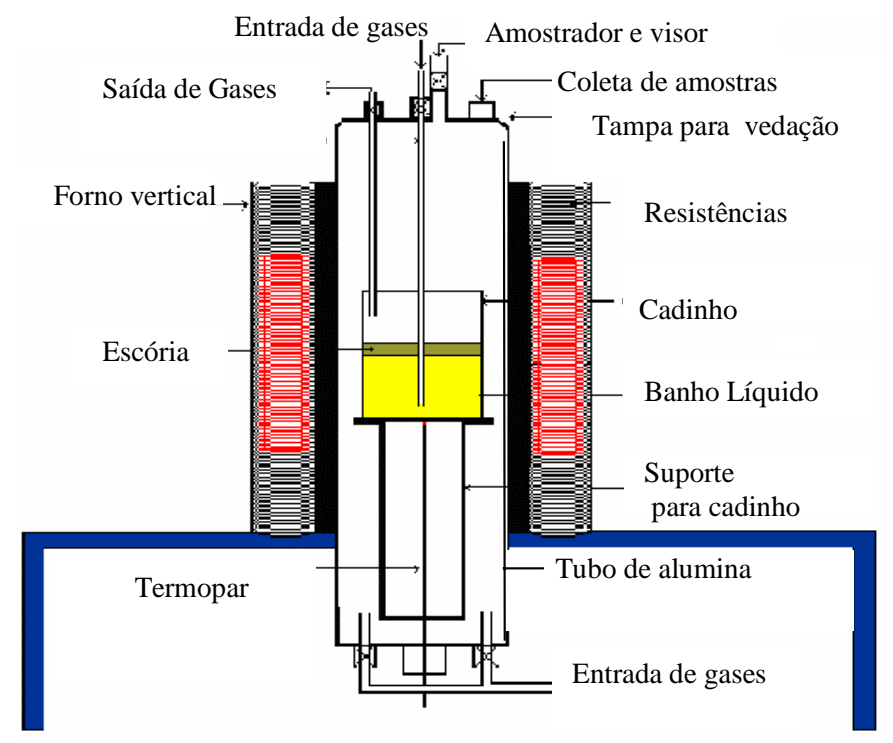

Figura 1 . Esquema do forno e câmara de reação. 
considerando a redução do $\mathrm{MnO}$ e não do $\mathrm{MnO}_{2}$.

Para reduzir todo o minério, a massa de carbono estequiométrico necessária foi de $37 \mathrm{~g}$, e foi usado o mesmo grafite do qual foram confeccionados os cadinhos como fonte de carbono.

As tabelas 2 e 3 , a seguir, mostram os ensaios feitos para estudar a influência da temperatura e do tamanho das partículas de grafite na velocidade de redução do MnO pelo carbono sólido.

Tabela 2. Ensaios realizados para estudar a influência da temperatura na cinética de redução do $\mathrm{MnO}_{2}$ pelo carbono sólido.

\begin{tabular}{cccc}
\hline Ensaio & $\begin{array}{c}\text { Massa do } \\
\text { Grafite[g] }\end{array}$ & $\begin{array}{c}\text { Massa do } \\
\text { minério[g] }\end{array}$ & $\begin{array}{c}\text { Tempera- } \\
\text { tura }\left[{ }^{\circ} \mathbf{C}\right]\end{array}$ \\
\hline$(1)$ & & & 1600 \\
$(2)$ & 37 & 150 & 1550 \\
$(3)$ & & & 1500 \\
\hline
\end{tabular}

Tabela 3. Ensaios realizados para estudar a influência do tamanho das partículas de grafite na velocidade de redução do $\mathrm{MnO}_{2}$ pelo carbono sólido a $1600{ }^{\circ} \mathrm{C}$.

\begin{tabular}{cccc}
\hline Ensaio & $\begin{array}{c}\text { Massa do } \\
\text { Grafite[g] }\end{array}$ & $\begin{array}{c}\text { Granulo- } \\
\text { metria[mm] }\end{array}$ & $\begin{array}{c}\text { Massa do } \\
\text { Minério[g] }\end{array}$ \\
\hline$(1)$ & 37 & 0,230 & 150 \\
$(2)$ & & 0,162 & \\
$(3)$ & & 0,057 & \\
\hline
\end{tabular}

\section{RESULTADOS E DISCUSSÃO}

A partir das análises químicas realizadas nas amostras de metal, foram calculadas as concentrações de $\mathrm{Mn}$ no banho, em $\mathrm{mol} / \mathrm{cm}^{3}$, usando a seguinte equação:

$$
C_{M n}=\frac{n M n}{V}
$$

Onde $C_{M n}$ é a concentração de Mn dado em $\left(\mathrm{mol} / \mathrm{cm}^{3}\right), n M n$ é o número de moles de manganês no banho e $V$ o volume do banho em $\left(\mathrm{cm}^{3}\right)$.

Para calcular o volume do banho, nos instantes das amostragens, considerouse a massa das amostras retiradas (3g) e o aumento da porcentagem de carbono no banho, devido ao aumento da quantidade de Mn. A variação da quantidade de silício no banho foi muito pequena $(0,2 \%$ a $0,3 \%)$, e nem todas as amostras apresentaram silício. Assim sendo, foi desprezado o silício na variação de volume.

Com os valores de concentração de $\mathrm{Mn}$ obtidos, foram traçadas curvas de variação de concentração com o tempo.

Os valores das velocidades de redução do $\mathrm{MnO}(\mathbf{V r})$, foram determinados por método gráfico computacional, pelo programa MICROCAL ORIGIN 5.0. Para isto, foram traçadas curvas de variação do número de moles de $\mathrm{Mn}$ no banho com o tempo. O programa utilizado forneceu então os valores das velocidades tomando a tangente desta curva em cada ponto de amostragem; sendo a velocidade obtida no instante zero, considerada a velocidade inicial do processo (Vi).

Portanto, os valores obtidos para a velocidade de redução do $\mathrm{MnO}$, ou de incorporação de manganês ao banho, foram expressos em: Vr e $\mathrm{Vi}=\mathrm{mol} / \mathrm{min}$.

\subsection{Influência da temperatura}

A tabela 4 mostra a variação da porcentagem de manganês no banho para os ensaios realizados para estudar a influência da temperatura, na cinética de redução do $\mathrm{MnO}$ usando minério.

Tabela 4. Variação da porcentagem de Mn com o tempo em diferentes temperaturas na

granulometria do grafite de $0,162 \mathrm{~mm}$.

\begin{tabular}{cccc}
\hline $\begin{array}{c}\text { Tempo } \\
\text { [min] }\end{array}$ & $\mathbf{1 6 0 0}^{\circ} \mathbf{C}$ & $\mathbf{1 5 5 0}^{\circ} \mathbf{C}$ & $\mathbf{1 5 0 0}^{\circ} \mathbf{C}$ \\
\hline 0 & 56,6 & 56,6 & 56,6 \\
5 & 59,9 & 58,6 & 57,7 \\
10 & 61,8 & 60,1 & 59,0 \\
15 & 62,6 & 60,7 & 59,3 \\
20 & 63,0 & 61,0 & 59,5 \\
25 & 63,3 & 61,3 & 59,5 \\
30 & 63,4 & 61,3 & 59,6 \\
\hline
\end{tabular}

O banho inicial foi preparado com $60 \%$ de $\mathrm{Mn}$, porém como todo $\mathrm{Fe}_{2} \mathrm{O}_{3}$ foi reduzido antes de 5 minutos houve um 
aumento de 12 gramas na massa do banho, diminuindo o teor de $\mathrm{Mn}$ para 56,6\%. O teor de $\mathrm{FeO}$ foi determinado pela análise química de uma amostra que foi retirada aos 4 minutos de ensaio e foi igual a 0,1\%. Portanto foi considerado que todo $\mathrm{FeO}$ foi reduzido antes de 5 minutos. Foi também considerado, como citado anteriormente, que todo $\mathrm{MnO}_{2}$ foi reduzido a $\mathrm{MnO}$, de acordo com os trabalhos realizados por Akdogan e Eric [19] e Rankin e Wynnyckyj [20]. Por isto todo tratamento foi realizado considerando a redução do $\mathrm{MnO}$ e não do $\mathrm{MnO}_{2}$.

As curvas de variação da concentração de Mn no banho em função do tempo são mostradas na figura 2 .

Os valores das concentrações de $\mathrm{Mn}$, nos tempos de amostragem, e as respectivas velocidades de redução, são mostrados, a seguir, na tabela 5 .

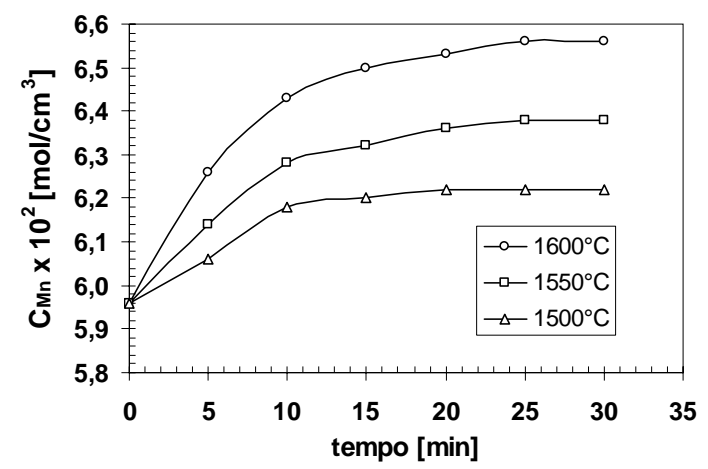

Figura 2. Variação da concentração de Mn no banho, para diferentes temperaturas.

Através de um gráfico ln Vi x $1 / \mathrm{T}$, foi calculada a energia de ativação aparente da reação, o que é mostrado na figura 3. O valor da energia de ativação aparente encontrado é mostrado na tabela 6 .

Pela análise da tabela 5 e da figura 3 nota-se que a velocidade de redução do $\mathrm{MnO}_{2}$, aumenta com o aumento da temperatura.

Tabela 5. Variação da concentração de Mn no banho, e as respectivas velocidades de redução de $\mathrm{MnO}$ nos ensaios feitos com diferentes temperaturas

\begin{tabular}{|c|c|c|}
\hline $\begin{array}{c}\text { Tempo } \\
\text { [min] }\end{array}$ & $\begin{array}{c}\mathrm{C.10}^{2} \\
{\left[\mathrm{mols} / \mathrm{cm}^{3}\right]}\end{array}$ & $\begin{array}{c}\text { Vr.10 } \\
{[\mathrm{mols} / \mathrm{min}]}\end{array}$ \\
\hline \multicolumn{3}{|c|}{$1600^{\circ} \mathrm{C}$} \\
\hline 0 & 5,96 & 6,30 \\
\hline 5 & 6,26 & 4,42 \\
\hline 10 & 6,43 & 2,18 \\
\hline 15 & 6,50 & 1,40 \\
\hline 20 & 6,53 & 8,19 \\
\hline 25 & 6,56 & 4,19 \\
\hline 30 & 6,56 & 1,82 \\
\hline \multicolumn{3}{|c|}{$1550^{\circ} \mathrm{C}$} \\
\hline 0 & 5,96 & 3,82 \\
\hline 5 & 6,14 & 2,62 \\
\hline 10 & 6,28 & 1,26 \\
\hline 15 & 6,32 & 9,28 \\
\hline 20 & 6,36 & 6,19 \\
\hline 25 & 6,38 & 0,291 \\
\hline 30 & 6,38 & 0,11 \\
\hline \multicolumn{3}{|c|}{$1500^{\circ} \mathrm{C}$} \\
\hline 0 & 5,96 & 2,04 \\
\hline 5 & 6,06 & 1,51 \\
\hline 10 & 6,18 & 7,64 \\
\hline 15 & 6,20 & 4,19 \\
\hline 20 & 6,22 & 0,22 \\
\hline 25 & 6,22 & 0,11 \\
\hline 30 & 6,22 & 0,073 \\
\hline
\end{tabular}

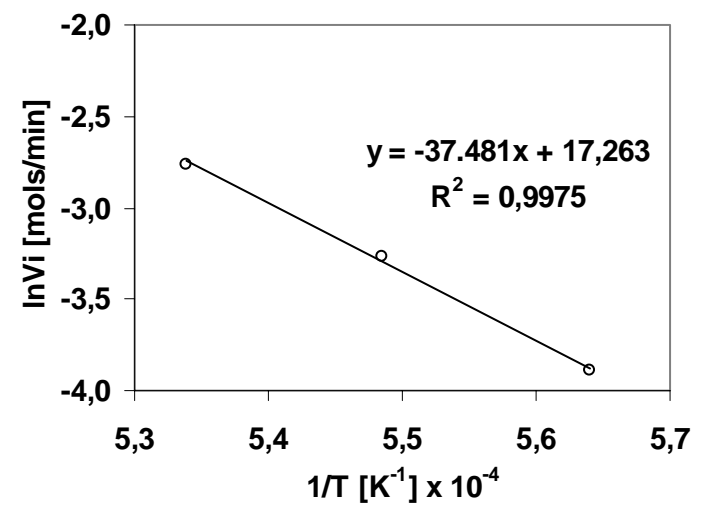

Figura 3. Determinação da energia de ativação aparente.

Tabela 6. Valor da energia de ativação aparente encontrado.

\begin{tabular}{cc}
\hline ensaios & $\mathbf{E}[\mathbf{k J} / \mathbf{m o l}]$ \\
\hline 1,2 e 3 & 311,3 \\
\hline
\end{tabular}

Estes resultados são coerentes com os encontrados por com Rankin e Wynnyckyj [20] que determinaram os valores da energia de ativação para que para redução do $\mathrm{MnO}$ pelo grafite na faixa 
de temperatura de $1227^{\circ} \mathrm{C}$ a $1427^{\circ} \mathrm{C}$. Os valores encontrados de energia de ativação aparente foram na faixa de 240 a $257 \mathrm{~kJ} / \mathrm{mol}$ que segundo os autores são característicos de processos de redução controlados pela reação de Boudouard $\left(\mathrm{CO}_{2}+\mathrm{C}_{(\mathrm{s})}=2 \mathrm{CO}_{(\mathrm{g})}\right)$. Este fato é confirmado por ensaios realizados pelos autores, onde o tamanho das partículas de $\mathrm{MnO}$ foi variado e a velocidade de redução não foi alterada, o que faz com que a possibilidade da a etapa controladora ser a reação de redução do $\mathrm{MnO}$ seja descartada. Comprando o valor de energia de ativação aparente encontrado no presente trabalho de $311,3 \mathrm{~kJ} / \mathrm{mol}$, com os resultados encontrados Rankin e Wynnyckyj, pode-se afirmar que a redução do minério de manganês pelo carbono sólido é controlado pela reação de Boudouard.

Baseado nestes fatos, e que a escória estará sempre líquida nas temperaturas usadas [21], pode-se considerar que a as reações de redução que ocorrem no processo são [22, 24]:

$$
\begin{aligned}
& 2 \mathrm{MnO}_{2}+2 \mathrm{CO}_{(\mathrm{g})}=2(\mathrm{MnO})+2 \mathrm{CO}_{2(\mathrm{~g})} \ldots . \\
& (\mathrm{MnO})+\mathrm{CO}_{(\mathrm{g})}=\mathrm{Mn}_{(\mathrm{l})}+\mathrm{CO}_{2(\mathrm{~g})} \\
& \mathrm{CO}_{2}+\mathrm{C}_{(\mathrm{s})}=2 \mathrm{CO}_{(\mathrm{g})}
\end{aligned}
$$

Os resultados também estão de acordo com os encontrados por Akdogan e Erick [19], que estudaram a redução do $\mathrm{MnO}$ pelo carbono sólido em diferentes minérios, na faixa de temperatura entre $1100^{\circ} \mathrm{C}$ e $1350^{\circ} \mathrm{C}$. Os autores afirmam que a velocidade de redução do $\mathrm{MnO}$, aumenta com o aumento da temperatura.

\subsection{Influência da granulometria do grafite}

A tabela 7 mostra a variação da porcentagem de manganês no banho para os ensaios.

Os valores das concentrações de Mn nos tempos de amostragem e as respectivas velocidades de redução são mostrados na tabela 8.
Tabela 7. Variação da porcentagem de Mn com o tempo nas reações de redução do $\mathrm{MnO} 2$ para diferentes granulometrias de carbono a $1600^{\circ} \mathrm{C}$.

\begin{tabular}{cccc}
\hline $\begin{array}{c}\text { tempo } \\
\text { [min] }\end{array}$ & $\mathbf{0 , 2 3 0 ~} \mathbf{~ m m}$ & $\mathbf{0 , 1 6 2} \mathbf{~ m m}$ & $\mathbf{0 , 0 5 7 m m}$ \\
\hline 0 & 56,6 & 56,6 & 56,6 \\
5 & 59,5 & 59,9 & 60,7 \\
10 & 61,4 & 61,8 & 62,9 \\
15 & 62,2 & 62,6 & 63,8 \\
20 & 62,6 & 63,0 & 64,0 \\
25 & 63,0 & 63,3 & 64,2 \\
30 & 63,1 & 63,4 & 64,3 \\
\hline
\end{tabular}

Pela análise da tabela 8 e da figura 4 nota-se que a velocidade de redução do $\mathrm{MnO}$ aumenta com a diminuição do tamanho das partículas de grafite usadas.

Tabela 8. Variação da concentração de Mn no banho, e as respectivas velocidades de redução de nos ensaios variando o tamanho

\begin{tabular}{ccc}
\multicolumn{3}{c}{ das partículas de grafite. } \\
$\begin{array}{c}\text { tempo } \\
\text { [min] }\end{array}$ & $\begin{array}{c}\mathbf{C . 1 0 ^ { 2 }} \\
{\left[\mathbf{m o l s} / \mathbf{c m}^{3}\right]}\end{array}$ & $\begin{array}{c}\text { Vr.10 }^{\mathbf{2}} \\
\text { [mols/min] }\end{array}$ \\
\hline \multicolumn{3}{c}{$\mathrm{d} 1=0,057 \mathrm{~mm}$} \\
\hline 0 & 5,96 & 8,74 \\
5 & 6,32 & 5,64 \\
10 & 6,52 & 2,31 \\
15 & 6,59 & 1,29 \\
20 & 6,61 & 0,47 \\
25 & 6,63 & 0,36 \\
30 & 6,64 & 0,29 \\
\hline \multicolumn{3}{c}{$\mathrm{d} 2=0,162 \mathrm{~mm}$} \\
0 & 5,96 & 6,30 \\
5 & 6,26 & 4,42 \\
10 & 6,43 & 2,18 \\
15 & 6,50 & 1,40 \\
20 & 6,53 & 0,819 \\
25 & 6,56 & 0,419 \\
30 & 6,57 & 0,182 \\
\hline \multicolumn{3}{c}{$\mathrm{d} 3=0,230 \mathrm{~mm}$} \\
\hline 0 & 5,96 & 5,53 \\
5 & 6,22 & 3,95 \\
10 & 6,39 & 2,02 \\
15 & 6,45 & 1,38 \\
20 & 6,50 & 1,00 \\
25 & 6,54 & 0,73 \\
30 & 6,22 & 0,073 \\
\hline
\end{tabular}

A Figura 4 mostra as curvas com a variação da concentração do $\mathrm{Mn}$ com o tempo, para diferentes tamanhos de partículas de grafite. 


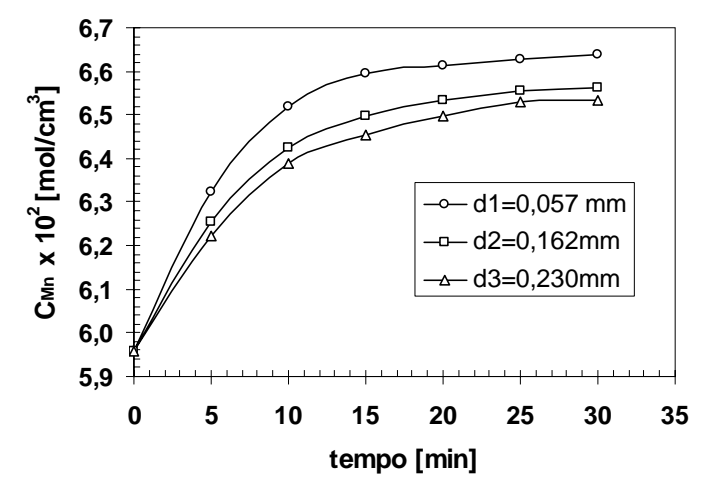

Figura 4. Variação de concentração de Mn para diferentes granulomteria de grafite $1600^{\circ} \mathrm{C}$.

Estes resultados são compatíveis com os encontrados por Akdogan e Erick [19]. Os autores estudaram a influência do diâmetro das partículas de grafite na velocidade de redução de um minério de manganês e observaram que há uma diminuição na velocidade de redução, quando as partículas do redutor aumentam de 0,044 mm, para tamanhos entre 0,150 e $0,210 \mathrm{~mm}$

No presente trabalho o maior aumento na velocidade de redução do $\mathrm{MnO}$ encontrada (1,39 vezes), foi quando houve uma diminuição no tamanho das partículas de grafite de 0,162 para $0,057 \mathrm{~mm}(2,84$ vezes). Quando se diminuiu o tamanho de 0,230 para $0,162 \mathrm{~mm}$ (1,42 vezes) a variação foi menor (1,14 vezes). Este fato pode ser explicado pela análise da tabela 9.

Tabela 9. Variação da área de contato entre escória e grafite para diferentes tamanhos e massas de partículas de grafite de

\begin{tabular}{|c|c|c|}
\hline \multirow[t]{2}{*}{$\begin{array}{c}\text { Diâmetro } \\
\text { [cm] }\end{array}$} & \multicolumn{2}{|c|}{$\begin{array}{c}\text { Área de Contato } \\
{\left[\mathrm{cm}^{2}\right]}\end{array}$} \\
\hline & $14 \mathrm{~g}$ & $37 \mathrm{~g}$ \\
\hline 0,0230 & 2028,9 & 5362,3 \\
\hline 0,0162 & 2880,65 & 7613,2 \\
\hline 0,0057 & 8187,1 & 21637,4 \\
\hline
\end{tabular}

A tabela 9 mostra que o aumento na área de contato entre escória grafite é aproximadamente de 4 vezes para a diminuição dos tamanhos das partículas de $0,057 \mathrm{~mm}$ para $0,162 \mathrm{~mm}$; e de 1,4 vezes para a diminuição de $0,162 \mathrm{~mm}$ para ENGEVISTA, v. 10, n. 2, p. 82-90 dezembro 2008 0,230mm. Esta variação justifica a maior velocidade encontrada no primeiro caso.

\section{5- CONCLUSÕES}

1. Uma diminuição na granulometria do grafite, aumenta a velocidade de redução do $\mathrm{MnO}$, devido a um aumento na área de contato entre a escória e o grafite

2. A velocidade de redução do $\mathrm{MnO}$ pelo carbono sólido aumenta com o aumento da temperatura, e entre $1500^{\circ} \mathrm{C}$ e $1600^{\circ} \mathrm{C}$ ocorre um aumento de 2,8 a 3,2 vezes nas velocidades iniciais.

3. A energia de ativação aparente obtida para a redução do $\mathrm{MnO}$ pelo carbono sólido é de $311,3 \mathrm{~kJ} / \mathrm{mol}$.

4. A redução do minério de manganês pelo carbono sólido é controlado pela reação de Boudouard.

5. A área de contato entre o redutor e o $\mathrm{MnO}$, é um fator preponderante na redução do $\mathrm{MnO}$, seja pelo carbono sólido

\section{6-REFERÊNCIAS BIBLIOGRÁFICAS}

[1] PRAKASH, S. Smelting reduction of prereduced iron ore. Ironmaking and Steelmaking, pp. $468-475$, vol. 24, $n^{\circ} .6$, 1997.

[2] PRASAD,K.K. History of Developments in Smelting Reduction. Metal News, pp. 2-6, june, 1996.

[3] DAS, S.N. ; PAL, P.K. Smelting Reduction Processes Na Overview. Metal News, pp.7-14, april, 1994.

[4] OLIVEIRA, J.R. ; TENÓRIO, J.A.S.; ASSIS, P.S. Smelting reduction: Uma alternativa para a produção de gusa,aço e ferro-ligas. METALURGIA E MATERIAIS, 54. ABM. pp.580-581. 1998.

[5] OLIVEIRA, J.R. ; TENÓRIO, J.A.S.; MOURÃO,M.B. Análise do Processo de Fabricação de Aço pela Técnica de Fusão 
Redução. $48^{\circ}$ Congresso Anual da Associação Brasileira de Metalurgia e Materiais. Resumos. Rio de Janeiro, jul. A.B.M., pp.193-194. 1993.

[6] FRUEHAN, R.J. Reactions Rates and Rate Limiting Factors in Iron Bath Smelting. Savard/Lee International Symposium on Bath Smelting, TMS, pp. 233-248, october. 1992.

[7] FRUEHAN, R.J. Iron Bath Smelting Current Status and Understanding. International Conference of New Smeltig Reduction and Near Shape Casting Technologies for Steel, pp.39-56, 1990.

[8] FRUEHAN, R.J.Analysis of Bath Smelting Processes for Producing Iron. ISIJ International, v.32, n.4, pp.538-544, 1992.

[9] KATAYAMA, H. et al. Reactions and Heat Transfer in Bath Smelting of Iron and Ferroalloys. Savard/Lee International Symposium on Bath Smelting, TMS, pp. 431-444, october. 1992.

[10] KATAYAMA, H. et al. The Characteristics and the Function of a Thick Slag Layer in the Smelting Reduction Process. ISIJ International, v.33, n.1, pp.124-132, 1993.

[11] OETERS, F. Fundamentals of InBath Smelting with Post-Combustion, Sarvard/Lee International Symposium on Bath Smelting, TMS, 1992.

[12] IBARAKI, T.Development of Smelting Reduction of Iron Ore - An Approach to Commercial Ironmaking. Ironmaking Conference Proceedings, pp.95-102, 1989.

[13] TANABE, $H$. et al. The Primary Stage of the Development of Smelting Reduction Process in NKK. Ironmaking Conference Proceedings, pp.89-94, 1989.

[14] KITAGAWA, T. et al. Operational Results of 100 ton/ day Test Plant for Smelting Reduction Iron Ore in NKK. Savard/Lee International Symposium on
Bath Smelting, TMS, pp.611-622, october. 1992.

[15] KATAYAMA, H. Reactions and Heat Transfer in Bath Smelting of Iron and Ferroalloys. Savard/Lee International Symposium on Bath Smelting, TMS, pp. 431-444, october. 1992.

[16] KATAYAMA,H. al.The Characteristics and the Function of a Thick Slag Layer in the Smelting ReductionProcess.ISIJ nternational, v.33, n.1, pp.124-132, 1993.

[17] KUANGDI, X. et al. The Kinetics of Reduction of $\mathrm{MnO}$ in Molten Slag with carbon Satured Líquid Iron. ISIJ International, v.33, n.1, pp.104 -108, 1993.

[18] OLIVEIRA, J.R. Redução do $\mathrm{MnO}$ Pelo Carbono Dissolvido no Banho, e Pelo Carbono Sólido na Escória. Tese de Doutorado Apresentado à, Universidade de São Paulo, São Paulo, 2001.

[19] AKDOGAN, G. ; ERICK, R.H. Kinetics of the Solid-State Carbothermic Recuction of Wessel Manganese Ores. Metallurgical and Materials Transactions B, pp. 13 -24, february, 1995.

[20] RANKIN, W.J. ; WYNNYCKCKYJ, J.R. Kinetics of Reduction of $\mathrm{MnO}$ in Powder Mixtures with Carbon. Metallurgical and Materials Trasactions B, v.28B, april, pp. 307-319, 1997.

[21] SLAG ATLAS. Verlag Sthleisen M.V.H. Düsseldorf, 1981, pp.61.

[22] HOOPER, R.P. The Optimum utilization of Raw Materials in Manganese Smelting, Proceedings of Infacon, Published by South Africa Institute of Mining and Metalurgy, Joanesburg, South Africa, pp. 101 -105, 1975.

[23] SILVEIRA, R.C. Contribuição ao Estudo Elétrico e Metalúrgico dos Fornos Elétricos de Redução. Belo Horizonte, Tese de Livre Docência, UFMG, 61 pp. 1974. 
[24] CETEC - Fundação Centro Tecnológico de Minas Gerais, Analise Técnico Econômica dos processos de Produção de Ferro Manganês no AltoForno, Fornos Elétricos Abertos e Fornos Elétricos Fechados, Relatório Final Apresentado à Secretaria de Tecnologia Industrial do MIC, pp. 1-182, julho de 76. 